\title{
Parasite sources and sinks in a patched Ross-Macdonald malaria model with human and mosquito movement: implications for control
}

\author{
Nick W. Ruktanonchai*, David L. Smithł and Patrick De Leenheer ${ }^{\ddagger}$
}

July 14, 2016

\begin{abstract}
We consider the dynamics of a mosquito-transmitted pathogen in a multipatch Ross-Macdonald malaria model with mobile human hosts, mobile vectors, and a heterogeneous environment. We show the existence of a globally stable steady state, and a threshold that determines whether a pathogen is either absent from all patches, or endemic and present at some level in all patches. Each patch is characterized by a local basic reproduction number, whose value predicts whether the disease is cleared or not when the patch is isolated: patches are known as "demographic sinks" if they have a local basic reproduction number less than one, and hence would clear the disease if isolated; patches with a basic reproduction number above one would sustain endemic infection in isolation, and become "demographic sources" of parasites when connected to other patches. Sources are also considered focal areas of transmission for the larger landscape, as they export excess parasites to other areas and can sustain parasite populations. We show how to determine the various basic reproduction numbers from steady state estimates in the patched network and knowledge of additional model parameters, hereby identifying parasite sources in the process. This is useful in the context of control of the infection on natural landscapes, because a commonly suggested strategy is to target focal areas, in order to make their corresponding basic reproduction numbers less than one, effectively turning them into sinks. We show that this is indeed a successful control strategy -albeit a conservative and possibly expensive one- in case either the human host, or the vector does not move. However, we also show that when both humans and vectors move, this strategy may fail, depending on the specific movement patterns exhibited by hosts and vectors.
\end{abstract}

\footnotetext{
*University of Southampton, nrukt00@gmail.com

$\dagger$ University of Oxford, david.smith@zoo.ox.ac.uk

${ }^{\ddagger}$ Department of Mathematics and Department of Integrative Biology, Oregon State University, deleenhp@math.oregonstate.edu. Partially supported by NSF DMS grant 1411853.
} 


\section{Introduction}

Malaria is a mosquito-borne disease caused by Plasmodium parasites, and is responsible for hundreds of thousands of deaths every year worldwide. The malaria parasite is found in red blood cells of an infected person, and transmission to humans occurs predominantly through mosquito bites, although according to the CDC [10] "it can also be transmitted through blood transfusion, organ transplant, the shared use of needles or syringes contaminated with blood, or from a mother to her unborn infant before or during delivery (congenital malaria)". Transmission depends on complex interactions between moving mosquito [19], and moving human [28, 35, 36] populations to facilitate parasite spread.

Human and mosquito populations are often spatially clustered [24], causing malaria risk to be heterogeneous across spatial scales [16, 6]. Host mediated parasite movement on these landscapes drives source-sink parasite dynamics which elimination programs must account for [9], as areas with enough transmission to sustain parasite populations locally will export excess parasites through host movement, known as transmission foci, supporting parasite populations in sink areas, or areas where parasites would not persist otherwise [9]. As transmission foci enable parasite persistence in sinks, they have been proposed as prime targets for control efforts $[9,27]$. Conceptually, regional malaria elimination can then be achieved by reducing transmission within all transmission foci to below self-sustaining levels [25].

Simply targeting the areas with the highest apparent transmission neglects human and mosquito movement and their role in parasite persistence, however, causing movement processes to potentially undermine elimination efforts [36]. Using a patched Ross-Macdonald model, we identify transmission foci in the context of both human and mosquito movement, and determine whether a strategy that targets foci exclusively is sufficient for parasite elimination, finding that while this strategy works when either humans or mosquitoes do not move between patches, there are network topologies where parasites persist even if transmission in all focal areas are brought to below sustainable levels.

The celebrated Ross-Macdonald model goes back to the groundbreaking work of Ronald Ross who received the Nobel Prize in Physiology or Medicine in 1902 for elucidating the complex infection cycle of malaria. Ross' model has since then been applied and refined by many authors including Macdonald, see [33, 34] for recent reviews. Classical Ross-Macdonald models consider infection dynamics in a single patch, but for all the reasons mentioned above, we extend this here to a patched model, a variant of which was first proposed and analyzed in [17]. Another variant of this model, considered in [30] and reviewed in [3, 37, 15], includes host movement, but excludes vector movement. More spatial models for malaria transmission have been reviewed in $[11,15]$ where space is treated discretely in the form of distinct patches, and in $[15,12]$ where space is either treated as discrete or as continuous, in the latter case leading to models that take the form of reaction-diffusion equations.

Models that include mobility need to specify how exactly vectors and humans move, and here we have adopted the so-called Lagrangian approach, see [11] and references therein. A salient feature of the Lagrangian model is that all individuals are declared to be residents of a specific patch, but that they can spend parts of their time in other patches, where they might infect others, or pick up the infection. 
This is in contrast to the more popular Eulerian approach, where individuals are not assigned to a particular patch, but instead simply move around between the various patches at certain prescribed rates. Examples of the Eulerian approach can be found in various contexts related to the spread of infectious diseases such as in $[1,2,5,11]$, and are not restricted to malaria. Our methods can be used to study similar patched Ross-Macdonald models based on the Eulerian approach, but to keep our analysis concise, we restrict ourselves to models based on the less frequently used Lagrangian approach. More sophisticated patch models have been proposed more recently. These models have been coupled to agent-based models to incorporate movement of the individual agents (both vectors and humans) in response to other environmental triggers such as temperature or rainfall, revealing fascinating patterns in the numerical simulations of these hybrid systems, see [23]. The main contributions of this paper are:

1. Establish the global dynamics of a patched Ross-Macdonald model, a variant of which was first investigated in [17] and reviewed in [11]. This model assumes an arbitrary number of patches between which both humans and mosquitoes are allowed to move. These movement patterns are quantified by matrices which express the fractions of time spent by residents of each patch in all other patches. A single real and positive quantity -the spectral radius of a matrix defined in terms of model parameters of all patches, as well as the movement matrices- determines the fate of the infection in the network: When this spectral radius is less than one, the infection is cleared. When it is larger than one, all solutions converge to a unique positive steady state and the infection globally persists in all the patches.

Although our proof is based on techniques that are similar to those used in [11] for a closely related model, we have decided to include a concise and self-contained proof in an Appendix here, for two main reasons. First, there are important differences between the modeling assumptions made in [17], and those considered here. Secondly, our proof relies on specific irreducibility properties of the matrices that encode vector and host mobility, and these conditions are different from those stated in [11], in a rather subtle way.

2. Identify local sinks and sources from steady state measurements of infected humans in the network. Each patch in the patched RossMacdonald model has its own transmission characteristics. In fact, to each patch we can associate a basic reproduction number, which would predict infection persistence or clearance in this patch if the patch were isolated. Since control measures are often aimed at lowering the reproduction numbers of those patches with the highest reproduction number values, an obvious first step is to determine, or at least estimate, the basic reproduction numbers of every patch with as little knowledge of model parameter values as possible. We show how to do this, based on the steady state measurements of infected humans in all the patches of the network. It turns out that only a limited number of model parameters is needed to achieve this, and we precisely state which ones these are.

3. Investigate how the patch reproduction numbers, in conjunction 
with host and vector mobility patterns, affect disease persistence or clearance in the network. We first consider the special cases where either only humans, or only mosquitoes move. If all patches are hotspots (respectively, sinks), then no matter what the mobility pattern of the moving host is, the disease persists in (respectively, is cleared from) the network. Thus, the control strategy that makes the reproduction number of every patch less than one, is guaranteed to clear the infection from the network, no matter what the mobility pattern of the moving host is. However, when there is a mix of hotspots and sinks in the network, this control strategy might be too conservative: For some mobility patterns the infection might be cleared without any intervention, although it may persist for others. This also indicates that in this case, an alternative control strategy -namely to intervene in the mobility patterns of the hosts- might be sufficient to clear the infection; and it may even be a cheaper one in certain cases, in particular when imposing travel restrictions is more cost-effective. We end by considering the general scenario in which both humans and mosquitoes move. A striking difference, compared to the cases where only one population moves, is that now the control strategy that makes the basic reproduction numbers less than one in all patches, may fail to clear the infection from the network. Failure or success depends on the mobility patterns of both humans and mosquitoes. Similarly, it may happen that in a network consisting of only sources, the infection is cleared by itself, without any control intervention at all. These results indicate that controlling a malaria infection in a network depends in a subtle way on the interplay between local transmission characteristics in the patches on the one hand, and the movement patterns of both hosts on the other.

The rest of this paper is organized as follows. In Section 2 we introduce the patched Ross-Macdonald model and discuss its global behavior. Two Appendices contain the proof of this result. In Section 2 we also propose a solution to the problem of determining the local reproduction numbers of all the patches based on steady state measurements. In Section 3 we investigate how patch characteristics, together with mobility patterns of vectors and human hosts, affect disease clearance or persistence in the network. Implications for control strategies aimed at clearing the infection from the network are considered here as well. Finally, we conclude this paper with some remarks in Section 4.

\section{Malaria models}

\subsection{Single patch}

The core model on which we later base our patched model, is a (rescaling of a) single patch Ross-Macdonald model proposed in [33], see also [11, 34] :

$$
\begin{aligned}
\dot{X} & =a b \mathrm{e}^{-\mu \tau} Y\left(\frac{H-X}{H}\right)-r X \\
\dot{Y} & =a c \frac{X}{H}(V-Y)-\mu Y
\end{aligned}
$$


This model represents the dynamics for the number of infected humans $X$, and the number of infected mosquitoes $Y$ in a total human population of $H$, and a total mosquito population of $V$ individuals. Individuals in both populations are assumed to be either susceptible, or infected. Hence, the number of susceptible humans is $H-X$, and the number of susceptible mosquitoes is $V-Y$. The other parameters in this model are:

1. $r$ is the recovery rate of infected humans, and $\mu$ is the death rate of mosquitoes, both having units of 1 /time.

2. $a$ is the biting rate of mosquitoes with units of number of humans bitten per mosquito and per unit of time.

3. $\tau$ is the incubation period (in units of time), i.e. the expected time that elapses between the moment a mosquito picks up the infection, and the moment it becomes infectious. When $\tau$ is non-negligible compared to the expected lifetime of a mosquito $1 / \mu$, it may happen that an infected mosquito dies before it becomes infectious. Thus, whereas $Y$ is the number of infected mosquitoes, $\mathrm{e}^{-\mu \tau} Y$ represents the number of infectious mosquitoes which are capable of infecting susceptible humans. This explains the appearance of the exponential factor in equation (1).

4. $b$ and $c$ represent the probability that a bite by an infectious mosquito infects a susceptible human, and the probability that a bite by a susceptible mosquito of an infected human is successful, respectively.

Clearly, the first term in (1) and in (2) represents the infection rate of susceptible humans, and of susceptible mosquitoes, and the remaining terms in these equations are the (human) recovery rate, and the (mosquito) death rate.

We scale $X$ and $Y$ :

$$
x=\frac{X}{H}, \quad y=\frac{Y}{V}
$$

and obtain the proportions of infected humans $x$ and of infected mosquitoes $y$. We also introduce the ratio of the total number of mosquitoes over the total number of humans:

$$
m=\frac{V}{H}
$$

and then the dynamics for the proportions $x$ and $y$ is given by:

$$
\begin{aligned}
\dot{x} & =m a b \mathrm{e}^{-\mu \tau} y(1-x)-r x \\
\dot{y} & =\operatorname{acx}(1-y)-\mu y
\end{aligned}
$$

Defining the basic reproduction number ${ }^{1}$ following [33]:

$$
\begin{aligned}
R_{0} & =\frac{m a^{2} b c \mathrm{e}^{-\mu \tau}}{r \mu} \\
& =\frac{m a b \alpha \mathrm{e}^{-\mu \tau}}{r}
\end{aligned}
$$

\footnotetext{
${ }^{1}$ Note that if one applies the procedure in [13] to calculate the basic reproduction number, one obtains the square root of the expression on the right-hand side of (7). Note also that no matter which definition one uses, the statement of Theorem 1 describing the global behavior of the system, remains the same.
} 
where

$$
\alpha=\frac{a c}{\mu}
$$

is the probability that a susceptible mosquito is infected during its life time. We see that $(5)-(6)$ has a unique steady state $(\bar{x}, \bar{y})$ :

$$
\bar{x}=\frac{R_{0}-1}{R_{0}+\alpha}, \quad \bar{y}=\frac{\alpha\left(R_{0}-1\right)}{(\alpha+1) R_{0}}
$$

in $(0,1)^{2}$ if and only if $R_{0}>1$. Note also that $(0,0)$ is always a steady state of $(1)-(2)$.

The following global result can be proved using standard phase plane techniques, see [20] for instance. Alternatively, one could exploit the monotonicity of the system, see [32], as well as the Appendix, for more on monotone systems:

Theorem 1. If $R_{0}<1$, then all solutions of $(5)-(6)$ converge to $(0,0)$. If $R_{0}>1$, then all positive solutions of $(5)-(6)$ converge to $(\bar{x}, \bar{y})$.

Estimating $R_{0}$. We now turn to the question of how to estimate the value of $R_{0}$, using steady state measurements of the fraction of infected humans only. It turns out that additional information is needed, but that Theorem 1 readily provides the answer:

1. If $R_{0}>1$, then estimating $R_{0}$ based on observing the steady state value $\bar{x}$, and the knowledge of $\alpha$, is possible by simply inverting (10):

$$
R_{0}=\frac{1+\alpha \bar{x}}{1-\bar{x}}
$$

2. But if $R_{0}<1$, then the observed steady state is $(0,0)$. In this case, the value of $R_{0}$ cannot be estimated by observing the $(0,0)$ steady state, even if $\alpha$ is known.

Control measures. To clear the infection, one must make $R_{0}$ less than 1 , and in view of formula (7) this may be achieved by lowering $m, a, b$ and $c$ (or $\alpha$ ), or increasing $r, \mu$ and $\tau$. Practical control strategies could include the use of screens, bednets and repellents (decreases $a$ ), drug treatment (increases $r$ ), use insecticides (increases $\mu$ and decreases $m$ ), vaccination (decreases $b$ ), larval source management (decreases $m$ ), and relocation of humans (decreases $m$ ).

\subsection{Multi-patch}

Suppose that there are $n$ patches and that in each patch the disease dynamics obeys the Ross-Macdonald model (1) - (2). To distinguish the heterogeneity among the patches we shall use subscripts $i$ for the state variable and the model parameters associated to patch $i$.

Assuming that both humans and mosquitoes move, possibly with different movement patterns, we investigate the following coupled model, a variant of which was 
proposed and analyzed in [17] and reviewed in [11], and which is called a Lagrangian model in contrast to the Eulerian models in for example $[1,2]$ :

$$
\begin{aligned}
\dot{X}_{i} & =\left(\sum_{j=1}^{n} p_{i j} a_{j} b_{j} \mathrm{e}^{-\mu_{j} \tau_{j}} Y_{j}\right)\left(\frac{H_{i}-X_{i}}{H_{i}}\right)-r_{i} X_{i} \\
\dot{Y}_{i} & =\left(\sum_{j=1}^{n} q_{i j} a_{j} c_{j} \frac{X_{j}}{H_{j}}\right)\left(V_{i}-Y_{i}\right)-\mu_{i} Y_{i},
\end{aligned}
$$

for all $i=1, \ldots, n$. The parameter $p_{i j}\left(q_{i j}\right)$ represents the fraction of time a human (mosquito) of patch $i$ spends in patch $j$. Thus, for all $i, j=1, \ldots, n$,

$$
p_{i j} \geq 0, q_{i j} \geq 0, \text { and } \sum_{k=1}^{n} p_{i k}=1, \sum_{k=1}^{n} q_{i k}=1,
$$

Note that the non-negative matrix $P(Q)$ whose $(i, j)$ th entry is $p_{i j}\left(q_{i j}\right)$ is rowstochastic, that is, the row sums of $P(Q)$ are all equal to 1.

The model conveys the following idea: All individuals, whether they are human or mosquitoes, are assigned a resident patch, but spend some proportion of their time in other patches. Susceptible individuals -again, both human and mosquitoescan be infected at a rate which is an average of the infection rates across patches, weighted by the proportion of the time they spend there. For example, human residents of patch $i$, spend a proportion of their time in patch $j$. Of these human residents of patch $i$, a fraction $\left(H_{i}-X_{i}\right) / H_{i}$ is susceptible, and if they end up spending time in patch $j$, they may be infected by infectious mosquitoes there at a rate that is proportional to the number of infectious mosquitoes in that patch, which is $\mathrm{e}^{-\mu_{j} \tau_{j}} Y_{j}$. This infection rate is also proportional to the biting rate $a_{j}$ in that patch, and to the probability that transmission is successful, i.e to $b_{j}$. A similar explanation can be given for the infection of susceptible mosquitoes that reside in patch $i$.

We scale each $X_{i}$ and $Y_{i}$ by the corresponding total number of humans and mosquitoes in that patch:

$$
x_{i}=\frac{X_{i}}{H_{i}}, \quad y_{i}=\frac{Y_{i}}{V_{i}}
$$

and defining the ratios:

$$
m_{i j}=\frac{V_{j}}{H_{i}}
$$

yields the dynamics of the proportions $x_{i}$ and $y_{i}$ in each patch:

$$
\begin{aligned}
& \dot{x}_{i}=\left(\sum_{j=1}^{n} p_{i j} m_{i j} a_{j} b_{j} \mathrm{e}^{-\mu_{j} \tau_{j}} y_{j}\right)\left(1-x_{i}\right)-r_{i} x_{i} \\
& \dot{y}_{i}=\left(\sum_{j=1}^{n} q_{i j} a_{j} c_{j} x_{j}\right)\left(1-y_{i}\right)-\mu_{i} y_{i},
\end{aligned}
$$

For patch $i$, we define two patch characteristics:

$$
R_{0}^{i}=\frac{m_{i i} a_{i}^{2} b_{i} c_{i} \mathrm{e}^{-\mu_{i} \tau_{i}}}{r_{i} \mu_{i}}=\frac{m_{i i} a_{i} b_{i} \alpha_{i} \mathrm{e}^{-\mu_{i} \tau_{i}}}{r_{i}} \text { and } \alpha_{i}=\frac{a_{i} c_{i}}{\mu_{i}} .
$$


and we say that patch $i$ is a sink if $R_{0}^{i}<1$, and a focal area of transmission (or source) if $R_{0}^{i}>1$.

We also introduce the parameter vector $\rho$ whose components $\rho_{i}$ are the ratios of the total human population in patch $i$ and the total human population in the first patch:

$$
\rho_{i}=\frac{H_{i}}{H_{1}}
$$

Then system (15) - (16) can be re-written as

$$
\begin{aligned}
& \dot{x}_{i}=\left(\sum_{j=1}^{n} \rho_{i}^{-1} p_{i j} \rho_{j} R_{0}^{j} \alpha_{j}^{-1} r_{j} y_{j}\right)\left(1-x_{i}\right)-r_{i} x_{i} \\
& \dot{y}_{i}=\left(\sum_{j=1}^{n} q_{i j} \alpha_{j} \mu_{j} x_{j}\right)\left(1-y_{i}\right)-\mu_{i} y_{i},
\end{aligned}
$$

We note that $[0,1]^{2 n}$ is a forward invariant set for system $(19)-(20)$, and that $(x, y)=(0,0)$ is always a steady state. In what follows we denote the spectral radius of any matrix $A$ by $R(A)$, defined as:

$$
\begin{aligned}
R(A) & :=\sup \{|\lambda| \mid \lambda \text { is an eigenvalue of } A\} \\
& =\lim _{n \rightarrow \infty}\left\|A^{n}\right\|^{1 / n}
\end{aligned}
$$

where in the latter, well-known formula by Gelfand, $\|A\|$ denotes any matrix norm. We use the notation $\operatorname{diag}(x)$ for any vector $x$ in $\mathbb{R}^{n}$ to denote the diagonal matrix having the components of the vector $x$ on its diagonal. By slightly abusing notation, we denote for given vectors $x$ and $y$ in $\mathbb{R}^{n}$, the vectors $x y$ and $x / y$ obtained by component-wise multiplication and division respectively, assuming that the latter are well-defined. Before stating our main result we introduce one more matrix:

$$
S=P \operatorname{diag}\left(R_{0}\right) D^{-1} Q D, \text { where } D=\operatorname{diag}((a c) /(r \rho))
$$

The following dichotomy states that the global dynamics of system (19) - (20) is entirely determined by the value of the spectral radius of the matrix $S$ :

Theorem 2. Assume that $P Q$ and $Q P$ are irreducible matrices.

If $R(S)<1$, then $(x, y)=(0,0)$ is the only steady state of $(19)-(20)$, and it is globally asymptotically stable.

If $R(S)>1$, then system (19) - (20) has exactly two steady states, namely $(x, y)=(0,0)$ and a positive $(\bar{x}, \bar{y})$ in $(0,1)^{2 n}$. In this case, all nonzero solutions converge to $(\bar{x}, \bar{y})$.

The proof is included it in the Appendix.

Comments on the irreducibility of $P Q$ and $Q P$.

Theorem 2 is proved under an irreducibility condition for the two matrix products of the row-stochastic mobility matrices of humans and vectors. 
1. First, what does irreducibility of $P Q$ and $Q P$ mean?

A particularly convenient definition of irreducibility of a non-negative matrix is in graphical terms, as it relates to a specific connectedness property of an associated graph [7]. Suppose that $Z$ is an $n \times n$ non-negative matrix. Construct a directed graph with $n$ nodes by drawing a directed edge from node $i$ to node $j$ if and only if $Z_{i j}>0$. The irreducibility of the matrix $Z$ is equivalent to the strong connectedness of the directed graph. The latter property means that for any pair of nodes $(i, j)$ of the graph, there must exist a directed path from node $i$ to node $j$, obtained by following directed edges; equivalently, there must exist a finite sequence $k_{1}, \ldots, k_{m}$ with $k_{1}=i$ and $k_{m}=j$, such that $Z_{k_{1} k_{2}} Z_{k_{2} k_{3}} \ldots Z_{k_{m-1} k_{m}}>0$.

We shall now use this graphical condition to understand what the irreducibility of the matrix $Q P$ means. Suppose that there is a single infected mosquito in patch $i$, in an otherwise entirely susceptible population of humans and mosquitoes in all patches. We ask

whether or not this single infected mosquito in patch $i$ has the potential to cause a secondary mosquito infection in patch $j$,

and claim that this is possible if and only if the non-negative $(j, i)$ th entry of the matrix $Q P$, is in fact positive:

$$
0<[Q P]_{j i}=\sum_{k=1}^{n} Q_{j k} P_{k i} .
$$

Indeed, the single infected mosquito in patch $i$ can infect a susceptible human resident in any patch $k$, while that human is visiting patch $i$. Susceptible humans in patch $k$ spend a proportion $P_{k i}$ of their time in patch $i$. Once the human resident of patch $k$ is infected by the mosquito, it can in turn infect a susceptible mosquito that is visiting patch $k$, but resides in patch $j$. Mosquito residents of patch $j$, spend a proportion $Q_{j k}$ of their time in patch $k$. Now summing over all possible patches $k$, shows that the original infected mosquito in patch $i$ can cause a secondary mosquito infection in patch $j$, provided that the expression in $(22)$, which represents the $(j, i)$ th entry of the product $Q P$, is positive, as claimed. Irreducibility of the matrix $Q P$ therefore means that a single infected mosquito resident in any patch, has the potential to cause a mosquito infection in any other patch later on, although the latter infection is no longer necessarily a secondary infection, but may occur through a finite number of consecutive mosquito-human-mosquito infections as described above.

A convenient way of checking irreducibility of $P Q$ and $Q P$ goes as follows. First, we construct a bipartite graph. Recall that a bipartite graph is a specific kind of graph having the property that the set of nodes can be partitioned in two disjoint sets of nodes, such that directed edges only go from a node in one set, to a node in the other set. Here we construct a bipartite graph with $2 n$ nodes, whose node set is partitioned as $\mathcal{N}_{1} U \mathcal{N}_{2}$, such that both $\mathcal{N}_{1}$ and $\mathcal{N}_{2}$ 
each have exactly $n$ nodes. When $P_{i j}>0$ we draw a directed edge from the $i$ th node of $\mathcal{N}_{1}$, to the $j$ th node of $\mathcal{N}_{2}$. Similarly, when $Q_{k l}>0$ we draw a directed edge from the $k$ th node of $\mathcal{N}_{2}$, to the $l$ th node of $\mathcal{N}_{1}$. This bipartite graphs captures very well that the disease cannot be transmitted directly from host to host, or from vector to vector, but must go from vector to host, or from host to vector. One can think of the $\mathcal{N}_{1}$ as a representation of the $n$ patches, from which weighted edges emanate that indicate the proportion of time, humans spend among the patches (the entries of the matrix $P$ ). Similarly, $\mathcal{N}_{2}$ represents the $n$ patches, but now the weighted edges indicate the proportion of time mosquitoes spend among the patches (the entries of the matrix $Q$ ). We will "collapse" this bipartite graph in two distinct ways, ending up with two new directed graphs. These resulting graphs each have exactly $n$ nodes, and irreducibility of $Q P$ and $P Q$ will be equivalent to strong connectedness of these two graphs. Specifically, $\mathcal{N}_{1}$ is the node set of the first directed graph, and has a directed edge from node $i$ to node $j$ if $[P Q]_{i j}>0$, or equivalently if the bipartite graph has a directed path with exactly 2 edges, emanating from the $i$ th node of $\mathcal{N}_{1}$, and ending in the $j$ th node of $\mathcal{N}_{1}$. Of course, by the very nature of the bipartite graph, such a path must necessarily pass through some node $k$ belonging to $\mathcal{N}_{2}$. In a similar fashion, a second directed graph can be constructed, but the node set of this second graph consists of the $n$ nodes that belong to $\mathcal{N}_{2}$. Finally, irreducibility of $P Q$ and $Q P$ is equivalent to the strong connectedness of the two directed graphs we have just obtained.

This discussion concerning the irreducibility of $P Q$ and $Q P$ also sheds light on the reason why Theorem 2 establishes that if $R(S)>1$, then the model has a unique, globally stable steady state with respect to which all non-zero solutions converge; this steady state represents a disease which is endemic in all patches, both for humans, as well as for mosquitoes. Indeed, for this to happen, the result should hold if the initial condition corresponds to the presence of a single infected mosquito, or a single infected human. In fact, these are typical initial conditions one encounters in practice. The discussion presented here, in conjunction with Theorem 2, shows that thanks to the irreducibility of both $P Q$ and $Q P$, this single infected individual can indeed cause the disease to spread to the entire network for both populations, provided that $R(S)>1$.

2. Note that irreducibility of $P$ and $Q$ does not imply irreducibility of their products, as can be seen by the following simple example:

$$
P=\left(\begin{array}{ll}
0 & 1 \\
1 & 0
\end{array}\right)=Q \text { is irreducible, but } P Q=\left(\begin{array}{ll}
1 & 0 \\
0 & 1
\end{array}\right)=Q P \text { is not. }
$$

Note also that (entry-wise) positivity of one of the matrices, is sufficient for irreducibility of the two products, because the product of a positive matrix, with a stochastic matrix is always positive.

On the other hand, irreducibility of both $P$ and $Q$ is not necessary for the irreducibility of $P Q$ and $Q P$. This is illustrated by two important special cases that we consider in more detail later, namely when only humans move, but mosquitoes don't, and vice versa. In this case, irreducibility of the mobility 
matrix associated to the moving species, humans or mosquitoes, suffice to conclude irreducibility of the matrices $P Q$ and $Q P$. For example, if humans move according to a pattern described by an irreducible mobility matrix $P$, and mosquitoes don't move (hence $Q=I$ ), then $P Q=P=Q P$, and the irreducibility of both products is clearly satisfied.

3. Irreducibility of $P Q$ does not necessarily imply irreducibility of $Q P$, as seen by the following example:

$$
\begin{gathered}
\text { For } P=\left(\begin{array}{ll}
1 & 0 \\
1 & 0
\end{array}\right) \text {, and } Q=\left(\begin{array}{cc}
1 / 2 & 1 / 2 \\
1 & 0
\end{array}\right) \\
P Q=\left(\begin{array}{ll}
1 / 2 & 1 / 2 \\
1 / 2 & 1 / 2
\end{array}\right) \text { is irreducible, but } Q P=\left(\begin{array}{ll}
1 & 0 \\
1 & 0
\end{array}\right) \text { is not. }
\end{gathered}
$$

This shows that one cannot drop the irreducibility condition of one of the products.

4. It is claimed in [11] (p.554 in Theorem 1), that [17] proved that the conclusions of Theorem 2 in this paper hold when both $P$ and $Q$ are irreducible (rather than $P Q$ and $Q P$ ). As we have seen above, irreducibility of $P$ and $Q$ does not imply irreducibility of $P Q$ and $Q P$; and conversely, irreducibility of $P Q$ and $Q P$ does not imply irreducibility of $P$ and $Q$. Moreover, a closer inspection of the model in [17] reveals that the setup of patch connectivity considered there, is different from the one considered here. Indeed, in [17], humans are not mobile, and reside on a set of $n$ patches; mosquitoes reside in a disjoint set of $m$ patches from which they can visit the patches where humans live to cause or pick up the infection. Therefore, there is only one mobility matrix (denoted as $\Gamma$ ), associated to mosquito movement. Furthermore, the crucial part in the proof in [17] (Theorem 7 on p. 47), where the global stability result of the equilibria is established, is not shown there explicitly, but instead attributed to a theorem in [21]. We note that [21] precedes the theory of monotone dynamical systems [32] which was pioneered by Morris Hirsch in the 1980-ies by almost a decade, and that [17] was published around the time monotone systems theory was being developed, but, perhaps not surprisingly, without using that theory.

Because we were unable to verify the above mentioned claim in [11] -caused by the subtleties related to the irreducibility properties of the matrices $P, Q, P Q$ and $Q P$ - and because the proof methods of [17] and [21] pre-date the by now well-established theory of monotone systems, we decided to include a selfcontained, yet concise proof of Theorem 2 in the Appendix to this paper. We do not claim any originality about the proof itself. Indeed, once the correct irreducibility condition is established, the proof of global stability is based on arguments that are quite standard nowadays thanks to the development of the theory of monotone systems. Our main purpose, however, is to clarify some of the confusion surrounding the role of the irreducibility conditions of the mobility matrices. 
Estimating $R_{0}$. Theorem 2 provides a dichotomy for the global behavior of the coupled system (19) - (20), depending on whether $R(S)$ is less than or larger than one. We now turn to the question of estimating the vector $R_{0}$, based on steady state measurements of the infected human populations in the various patches.

We assume throughout that the products of both mobility matrices, namely $P Q$ and $Q P$, are irreducible, and then Theorem 2 suggests the following:

1. If $R(S)<1$, then system (19) - (20) has only one globally stable steady state at $(x, y)=(0,0)$. Hence, we cannot estimate the vector $R_{0}$, based on the observation of this steady state. This is similar to what happened in the single patch case.

2. If $R(S)>1$, then the irreducibility of $P Q$ and $Q P$ guarantees that system $(19)-(20)$ has a unique steady state $(\bar{x}, \bar{y})$ in $(0,1)^{2 n}$ which attracts all nonzero solutions. In order to locate which patches are foci, and which are sinks, we ask the following question:

Given (an estimate of) $\bar{x}$, can we find (a corresponding estimate of) $R_{0}$ ?

It is reasonable to assume that at the very least, some estimate of $\bar{x}$, the vector consisting of the fraction of infected humans in all patches, is available. This data can be collected from counts of malaria cases in the local hospitals of each patch, and dividing this number by the total human population per patch. To tackle this question, we first express the steady state equations, where we let 1 be the $n$-vector consisting of ones:

$$
\begin{aligned}
\operatorname{diag}((1-\bar{x}) / \rho) P \operatorname{diag}(\rho r \bar{y} / \alpha) R_{0} & =\operatorname{diag}(r) \bar{x} \\
\operatorname{diag}(1-\bar{y}) Q \operatorname{diag}(\mu \alpha) \bar{x} & =\operatorname{diag}(\mu) \bar{y}
\end{aligned}
$$

We proceed in two steps:

Step 1: Express $\bar{y}$ in terms of $\bar{x}$. This can be achieved by solving the second equation for $\bar{y}$ :

$$
\bar{y}=\operatorname{diag}^{-1}(1+\bar{z}) \bar{z}, \text { where } \bar{z}:=\operatorname{diag}^{-1}(\mu) Q \operatorname{diag}(\mu \alpha) \bar{x}
$$

Indeed, the second steady state equation (24) is equivalent to $\operatorname{diag}^{-1}(1-\bar{y}) \bar{y}=$ $\bar{z}$. Since the scalar function $y \rightarrow y /(1-y)$ with $y$ in $(0,1)$ is increasing, hence invertible with inverse $z \rightarrow z /(1+z)$, the above relation (25) follows.

Note that the practical relevance of formula (25) is that it allows us to find the vector of fractions of infected mosquitoes in all patches -a vector which is probably difficult to measure directly- in terms of $\bar{x}$ whose estimate is more feasible. But it also requires additional knowledge of certain system parameters, namely the vectors $\mu$ and $\alpha$, and the matrix $Q$ associated to mosquito movement. The latter matrix in particular, may be difficult to estimate.

Step 2: Solve for $R_{0}$ in terms of $\bar{x}$. Note that (23) is a linear system in the unknown vector $R_{0}$. Its solution is:

$$
R_{0}=A^{-1} \operatorname{diag}(r) \bar{x}
$$


provided that the matrix:

$$
A:=\operatorname{diag}((1-\bar{x}) / \rho) P \operatorname{diag}(\rho r \bar{y} / \alpha), \quad \bar{y} \text { given by }(25)
$$

is invertible.

Since $(\bar{x}, \bar{y})$ belongs to $(0,1)^{2 n}$, invertibility of $A$ is clearly equivalent to invertibility of $P$. Thus, if $P$ is invertible, then (26) yields the vector of the basic reproduction numbers of all the patches. In particular, we can then immediately read off which of the patches are sources, and which are sinks. An interesting situation arises when some of the patches in the network are sinks. Indeed, in this case, our method, allows us to estimate their basic reproduction number (assuming that the disease persists in the network), something which would have been impossible if these sinks were isolated patches as shown before. One limitation of our method is that the matrix $P$ should be invertible, and this may not always be the case, as

$$
P=\frac{1}{n}\left(\begin{array}{cccc}
1 & 1 & \ldots & 1 \\
\vdots & \vdots & \ldots & \vdots \\
1 & 1 & \ldots & 1
\end{array}\right)
$$

shows. The structure of this matrix implies that humans of every patch divide their time equally among all patches.

Comments on estimating $R_{0}$. Let us examine which model parameters, or parameter combinations, should be known in order to evaluate the right hand side of (26), assuming that we have at least an estimate of $\bar{x}$. From Step 1, we need the vectors $\mu$ and $\alpha$, and the matrix $Q$, associated to mosquito movement. From Step 2 , we see that we also need the vector $r$, the vector $\rho$, and the matrix $P$, associated to human mobility, and this matrix should be invertible.

In summary, we need the:

1. recovery rate vector $r$, and the death rate vector $\mu .^{2}$

2. vector $\alpha$, which consists of the probabilities that a susceptible mosquito becomes infected over its entire lifetime, in all the patches.

3. the matrix $P$, which quantifies human movement.

4. the matrix $Q$, which quantifies mosquito movement.

5. vector $\rho$, consisting of the ratios of the total human populations in the various patches compared to the first patch.

6. the vector $\bar{x}$, consisting of the proportions of human infected individuals in the various patches.

\footnotetext{
${ }^{2}$ Actually, slightly less information is required. Indeed, it suffices that we know the ratios of the recovery rates, and the ratios of the death rates in the different patches. This follows from the fact that in (25) and in (26), there are factors $\operatorname{diag}(\mu)$ and $\operatorname{diag}^{-1}(\mu)$, and $\operatorname{diag}(r) \operatorname{and} \operatorname{diag}^{-1}(r)$ pre-and post-multiplying the matrix $Q$ and $P$ respectively.
} 
Let us compare this to the traditional estimation method of $R_{0}$, based on the original definition (17), which provides formulas for its entries in terms of various model parameters. This method requires for each patch $i$, the following information:

1. the recovery rate $r_{i}$, the death rate $\mu_{i}$.

2. the probability $\alpha_{i}$.

3. the biting rate $a_{i}$.

4. the probability $b_{i}$ that an infectious mosquito bite successfully infects a susceptible human.

5. the ratio of total number of mosquitoes and total number of humans $m_{i i}=$ $V_{i} / H_{i}$.

6. the incubation period $\tau_{i}$.

From both lists above, we see that the first two items of each method are the same, but the next four are different.

The parameter which is perhaps the most difficult one to determine for our estimation method (26) is $Q$, the mobility matrix associated to mosquito movement. This requires estimates of time spent by the mosquitoes among the various patches. On the other hand, in cases where the geographic scale of the patches is large, compared to typical distances traveled by mosquitoes, one may argue that $Q=I$. This expresses that mosquitoes are confined to their patch of residence. To a lesser extent, the mobility matrix $P$ associated to human movement, may sometimes be difficult to estimate, although mobile telephony data could be used for this purpose by tracking the movements of cell phone users.

The parameters which are the hardest to determine for the traditional method (17) are the ratios $m_{i i}$ of the total number of mosquitoes over the total number of humans in each patch. Although the total number of humans in each patch is likely to be well-known in many cases, this is far less likely in case of mosquitoes.

Finally, an important limitation of our method, compared to the traditional one, is that we require that $R(S)>1$, so that the model has a positive steady state $(\bar{x}, \bar{y})$ in $(0,1)^{2 n}$. In other words, our method requires the disease to be endemic, whereas the traditional method also works when the disease is not endemic.

\section{Bounds for $R(S)$ and implications for control}

In this Section we perform a closer examination of the spectral radius of the matrix $S$ defined in (21), because the value of this spectral radius determines whether or not the malaria infection persists in the patched network. We shall derive sharp upper and sharp lower bounds for this spectral radius in terms of the basic reproduction numbers of all the patches, and the mobility matrices of vectors and hosts. Similar bounds have been obtained for various epidemic models of non-vector borne diseases and using the Eulerian approach to model movement of individuals, see [18] for an SEIRP-model ( $\mathrm{P}$ represents the class of partially immune individuals), [14] for an 
SIS-model, and [4] for an SIR-model of a large metropolitan city and several satellite cities representing a suburban area.

Our analysis shall start with some special cases where either only humans move, or only mosquitoes. Later we turn to the general case where both move. We will see that there are profound differences between the first two scenarios on the one hand, and the third one on the other.

A key technical property that we shall use repeatedly in this context, is that the spectral radius is a non-decreasing function over the set of non-negative matrices, i.e.:

$$
0 \leq A \leq B \Rightarrow R(A) \leq R(B)
$$

Here, the notation $A \leq B$ means that the entries of $B$ are not smaller than the corresponding entries of $A$. For a proof of this fact, see [7].

Below we use the notation $x_{\min }=\min _{i}\left(x_{i}\right)$ and $x_{\max }=\max _{i}\left(x_{i}\right)$ for a given vector $x$ in $\mathbb{R}^{n}$.

\subsection{Only humans move}

When only humans move it follows that the matrix associated to the mobility of mosquitoes, is the identity matrix:

$$
Q=I
$$

In this case, the matrix $S$ simplifies to the matrix $S_{h}$, which is defined as:

$$
S_{h}=P \operatorname{diag}\left(R_{0}\right),
$$

and then we have the following bounds on the spectral radius of $S_{h}$ :

Theorem 3. Assume that (28) and (29) hold. Then:

$$
\left(R_{0}\right)_{\min } \leq R\left(S_{h}\right) \leq\left(R_{0}\right)_{\max }
$$

Moreover, these bounds are sharp in the sense that there exist row-stochastic matrices $P_{\min }$ and $P_{\max }$ such that:

$$
\rho\left(P_{\min } \operatorname{diag}\left(R_{0}\right)\right)=\left(R_{0}\right)_{\min } \text { and } \rho\left(P_{\max } \operatorname{diag}\left(R_{0}\right)\right)=\left(R_{0}\right)_{\max }
$$

Proof. Note that $0 \leq S_{h}=P \operatorname{diag}\left(R_{0}\right)$ implies that:

$$
0 \leq\left(R_{0}\right)_{\min } P \leq S_{h} \leq\left(R_{0}\right)_{\max } P
$$

and hence (30) follows from (27) and the fact that $R(P)=1$ (since $P$ is rowstochastic). To prove that the lower bound is achieved, take $P_{\min }$ as the matrix having exactly one column consisting of ones, namely the $i$ th column where $i$ is any index such that $R_{0}^{i}=\left(R_{0}\right)_{\min }$, and all other columns are zero vectors. A straightforward calculation then shows that $R\left(P_{\min } \operatorname{diag}\left(R_{0}\right)\right)=\left(R_{0}\right)_{\min }$. Similarly, to prove that the upper bound is achieved, set $P_{\max }$ as the matrix having exactly one column consisting of ones corresponding to an index $j$ for which $R_{0}^{j}=\left(R_{0}\right)_{\max }$, and all other columns consisting of zero vectors. This proves (31). 
From the point of view of malaria eradication in case only humans move, Theorem 3 , in combination with Theorem 2 , has several implications:

1. If all patches are foci, then the malaria infection will persist in the network, independently of network topology as encoded by the (human) mobility matrix $P$. Indeed, when all patches are transmission foci, then $\left(R_{0}\right)_{\min }>1$, and therefore $R\left(S_{h}\right)>1$ by (30). Theorem 2 then implies that the infection persists.

2. If all patches are sinks, then the malaria infection will be cleared from the network, independently of network topology as encoded by the (human) mobility matrix $P$. Indeed, when all patches are sinks, then $\left(R_{0}\right)_{\max }<1$, and therefore $R\left(S_{h}\right)<1$ by $(30)$. Theorem 2 then implies that the infection is cleared.

3. One control strategy is to identify all the foci (this can be achieved using the procedure outlined in the previous Section), and make their corresponding basic reproduction number less than one by suitable local control measures, described in the Section devoted to a single patch. This strategy is guaranteed to clear the infection, independently of the network topology as encoded by the matrix $P$.

4. The latter control strategy is probably rather conservative because it is aimed at disease clearance for all network topologies. In practice, one is confronted with a specific topology, and it is conceivable that to clear the infection, not all foci should necessarily be made into sinks by appropriate local control measures. To see that this can indeed happen, we consider a scenario with two patches in which one patch is a source, and the other is a sink. Depending on the network topology the infection may be cleared or may persist, highlighting the crucial role played by the matrix $P$. Assume 2 patches such that

$$
R_{0}=\left(\begin{array}{l}
3 / 2 \\
1 / 2
\end{array}\right)
$$

In other words, patch 1 is a transmission focus, and patch 2 is a sink. Let

$$
P_{1}=\left(\begin{array}{ll}
1 / 2 & 1 / 2 \\
1 / 4 & 3 / 4
\end{array}\right) \text { and } P_{2}=\left(\begin{array}{ll}
3 / 4 & 1 / 4 \\
1 / 2 & 1 / 2
\end{array}\right)
$$

Then setting $S_{1}=P_{1} \operatorname{diag}\left(R_{0}\right)$ and $S_{2}=P_{2} \operatorname{diag}\left(R_{0}\right)$, we have that:

$$
R\left(S_{1}\right) \approx 0.92<1, \text { but } R\left(S_{2}\right) \approx 1.22>1
$$

Thus, when human mobility is encoded by the matrix $P_{1}$, the infection is cleared. But if it is encoded by $P_{2}$, the infection persists.

5. Theorem 3 also suggests an alternative control strategy, namely to control the people's mobility pattern by modifying the matrix $P$, for instance by prohibiting travel between certain patches. Indeed, the proof of Theorem 3 shows that by making $P$ equal to (or at least approximately equal to) the matrix $P_{\text {min }}$, we can minimize $R\left(S_{h}\right)$. The biological interpretation of $P_{\min }$ is 
that all humans should spend $100 \%$ of their time in the patch having lowest reproduction number, a result which makes sense intuitively.

Yet another control strategy can be gleaned from (30) in Theorem 3: The strategy which relocates people between patches in an appropriate way. To sketch the main idea behind this strategy, consider for simplicity a scenario with 2 patches where

$$
R_{0}^{1}=\frac{V_{1}}{H_{1}} \frac{a_{1} b_{1} \alpha_{1} \mathrm{e}^{-\mu_{1} \tau_{1}}}{r_{1}} \text { and } R_{0}^{2}=\frac{V_{2}}{H_{2}} \frac{a_{2} b_{2} \alpha_{2} \mathrm{e}^{-\mu_{2} \tau_{1}}}{r_{2}}
$$

and such that

$$
R_{0}^{1}<1<R_{0}^{2} .
$$

Consequently, $\left(R_{0}\right)_{\max }=R_{0}^{2}>1$, and therefore the infection will persist in the two-patch system, at least for some human mobility matrices $P$. Control strategies based on relocation only, amount to keeping all parameters $V_{i}, a_{i}, b_{i}, \alpha_{i}, \mu_{i}, \tau_{i}$ and $r_{i}$ fixed for $i=1,2$, but allowing $H_{1}$ and $H_{2}$ to vary, as long as their sum remains constant. In this particular case, to clear the infection, we would seek to decrease $R_{0}^{2}$ below 1 , while maintaining $R_{0}^{1}$ below 1 as well. This may be achieved by increasing $H_{2}$ and decreasing $H_{1}$ by an equal amount. In practice, this means that human individuals would be relocated from patch 1 to patch 2 . The difficulty lies in the fact that although we can obviously always make $R_{0}^{2}$ less than 1 by an appropriate decrease in $H_{2}$, the corresponding increase in $H_{1}$ might push $R_{0}^{1}$ above 1 , in which case the relocation strategy will fail to clear the infection.

\subsection{Only mosquitoes move}

In this case, the matrix $P$ associated to human movement, is the identity matrix:

$$
P=I
$$

The matrix $S$ simplifies to the matrix $S_{m}$ which is defined as:

$$
S_{m}=\operatorname{diag}\left(R_{0}\right) D^{-1} Q D
$$

and then spectral radius of $S_{m}$ is bounded as follows:

Theorem 4. Assume that (32) and (33) hold. Then:

$$
\left(R_{0}\right)_{\min } \leq R\left(S_{m}\right) \leq\left(R_{0}\right)_{\max }
$$

Moreover, these bounds are sharp in the sense that there exist row-stochastic matrices $Q_{\min }$ and $Q_{\max }$ such that:

$$
\rho\left(\operatorname{diag}\left(R_{0}\right) D^{-1} Q_{\min } D\right)=\left(R_{0}\right)_{\min } \text { and } \rho\left(\operatorname{diag}\left(R_{0}\right) D^{-1} Q_{\max } D\right)=\left(R_{0}\right)_{\max }
$$

Proof. Since $R(A B)=R(B A)$ for any square matrices $A$ and $D$, it follows that $R\left(S_{m}\right)=R\left(\operatorname{diag}\left(R_{0}\right) D D^{-1} Q\right)=R\left(Q \operatorname{diag}\left(R_{0}\right)\right)$, and then the rest of the proof is similar to that of Theorem 3. 
All the remarks we made concerning disease control in case only humans move, remain valid here as well: simply replace the matrix $P$ by the matrix $Q$ in the discussion following Theorem 3. In particular, when all patches are focal areas, the disease persists, and when all patches are sinks, the disease is cleared, independent of the network topology associated to the mosquito movement matrix $Q$. Hence, a conservative control strategy is to make the reproduction numbers of all patches less then one, using local control measures described in the Section devoted to a single patch. When some patches are transmission foci but others are sinks, there exist mosquito mobility matrices $Q$ which give rise to disease persistence, but also matrices $Q$ giving rise to disease clearance. Finally, another possible control strategy, is to redistribute mosquitoes between various patches, similarly to the relocation strategy of humans described in the previous subsection. In practice this can be achieved by placing repellants in patches with high basic reproduction values, effectively reducing the total number of mosquitoes $V$ in those patches. However, these mosquitoes will move to other patches, where they in turn will increase the basic reproduction number. The failure or success of this strategy depends on whether or not the replaced mosquitoes push the basic reproduction values in their new home patches above 1 .

\subsection{Both humans and mosquitoes move}

This is the general case, where both $P$ and $Q$ are assumed to differ from the identity matrix. First, we define the positive vector $d$ as:

$$
d=(a c) /(r \rho) \text {. }
$$

Note that this implies that the diagonal matrix $D$ in (21) contains the components of $d$ on its diagonal: $D=\operatorname{diag}(d)$, and hence (21) can be re-written as:

$$
S=P \operatorname{diag}\left(R_{0} / d\right) Q \operatorname{diag}(d)
$$

Then we have:

Theorem 5. The spectral radius of $S$ is bounded as follows:

$$
d_{\min }\left(R_{0} / d\right)_{\min } \leq R(S) \leq d_{\max }\left(R_{0} / d\right)_{\max }
$$

Moreover, these bounds are sharp in the sense that there exist pairs of row-stochastic matrices $\left(P_{\min }, Q_{\min }\right)$ and $\left(P_{\max }, Q_{\max }\right)$ such that:

$$
\begin{gathered}
\rho\left(P_{\min } \operatorname{diag}\left(R_{0} / d\right) Q_{\min } \operatorname{diag}(d)\right)=d_{\min }\left(R_{0} / d\right)_{\min }, \text { and } \\
\rho\left(P_{\max } \operatorname{diag}\left(R_{0} / d\right) Q_{\max } \operatorname{diag}(d)\right)=d_{\max }\left(R_{0} / d\right)_{\max } .
\end{gathered}
$$

Proof. From (36) follows that:

$$
d_{\min }\left(R_{0} / d\right)_{\min } P Q \leq S \leq d_{\max }\left(R_{0} / d\right)_{\max } P Q,
$$

and therefore, upon taking the spectral radius of the matrices above, the fact that $R(P Q)=1$ (because the product of two row-stochastic matrices is row-stochastic, 
hence has spectral radius 1), (27) implies (37). To prove (38) and (39) we use a similar argument as in the proof of Theorem 3. For instance, to prove (38) we can take $P_{\min }$ to be a matrix having exactly one column consisting of ones, namely the $i$ th column corresponding to the minimal component of the vector $R_{0} / d$, and all other columns are zero vectors. Similarly for $Q_{\min }$ we take a matrix having exactly one column consisting of ones, namely the $j$ th column corresponding to the minimal component of the vector $d$, and all other columns are zero vectors. Then a straightforward calculation shows that (38) holds.

Although many of the remarks we made concerning disease control following Theorem 3 and Theorem 4, remain valid in the case that both humans and mosquitoes move, we point out some striking differences:

1. The conservative control strategy that made the basic reproduction numbers in all patches less than one using local control measures, no longer guarantees that the disease will be cleared from the network, independently of the movement matrices $P$ and $Q$. Indeed, although this strategy ensures that $\left(R_{0}\right)_{\max }<1$, it does not necessarily make $d_{\max }\left(R_{0} / d\right)_{\max }<1$. For example, in a 2 patch system with:

$$
R_{0}=\left(\begin{array}{l}
1 / 2 \\
1 / 4
\end{array}\right) \text { and } d=\left(\begin{array}{c}
1 / 4 \\
1
\end{array}\right)
$$

there holds that:

$$
1 / 2=\left(R_{0}\right)_{\max }<1<d_{\max }\left(R_{0} / d\right)_{\max }=\max ((1 / 2) /(1 / 4), 1 / 4)=2 .
$$

Consequently, Theorem 5 also says that there are in fact network topologies for human $(P)$ and mosquito $(Q)$ movement, such that the disease persists (because the upper bound for $R(S)$, which is 2, can be achieved), despite the fact that $\left(R_{0}\right)_{\max }<1$. In other words, contrary to what happened in the cases where only humans or only mosquito move, the disease may persist in a network of sinks.

2. Similar arguments show that when both humans and mosquitoes move, it is possible that the disease is cleared from a network of sources. This contrasts the cases where either only humans, or only mosquitoes move. For instance, when

$$
R_{0}=\left(\begin{array}{l}
2 \\
4
\end{array}\right) \text { and } d=\left(\begin{array}{c}
1 \\
1 / 4
\end{array}\right)
$$

then

$$
1 / 2=(1 / 4) \min (2,16)=d_{\min }\left(R_{0} / d\right)_{\min }<1<\left(R_{0}\right)_{\min }=2 .
$$

The two examples above, indicate that knowledge of the maximal and minimal component of the vector $R_{0}$, i.e. the maximal and minimal basic reproduction number of all the patches in isolation, is no longer sufficient to predict disease clearance or persistence from the network. Instead, according to the bounds (37) in Theorem 5, the product of the maximal and minimal components of the vectors $d$ and $R_{0} / d$ are the relevant quantities. Therefore, control strategies focused on the basic reproduction numbers of isolated patches, are no longer adequate when both hosts and vectors move. From a practical control perspective, this may be the most important conclusion of the mathematical analysis presented here. 


\section{Conclusion}

Robust strategies for malaria elimination that account for parasite movement are critical for malaria control programs [25], and strategies that spatially target vector control and treatment will improve the efficiency of the use of limited resources. Being able to predict how control will affect parasite populations across networks of patches has been characterized statistically, but it also requires a mechanistic understanding of transmission and parasite mobility, as mediated by both mosquitoes and humans. However, most algorithms for quantifying transmission intensity across heterogeneous landscape either do not incorporate mobility in both hosts [29, 38] or are purely statistical identification methods [8]. In the multi-patch Ross-Macdonald model we analyzed to identify patches that are transmission foci, which incorporates both human and mosquito movement, we test whether targeting foci based on local estimates of transmission is a viable strategy for eliminating parasite populations regionally. We find that while this strategy is sufficient to eliminate all parasites if only humans or mosquitoes move, when both hosts move, there are network topologies that can cause a strategy that only targets foci to fail. This result highlights the complex interactions between malaria parasite, human, and mosquito populations caused by host mobility, and the need for understanding the specific movement patterns of humans and mosquitoes when developing malaria elimination strategies. More generally, it is well-known that the basic reproduction number $R_{0}$ plays an important role in various models of the spread of many infectious diseases, yet control measures aimed at simply reducing $R_{0}$ below 1 may be insufficient to clear the infection. Our results are in accordance with that observation.

We conclude with some comments related to the practical use of our results. A nontrivial problem when using the proposed patched Ross-Macdonald model, is to define the various patches in the system. Policy makers who would use this model in their decision process, will have to identify the various patches first, before they can implement specific control strategies. Obviously, there is no unique way to do this. For example, a lot depends on the geographic scale of the infection dynamics: this could range from systems of nearby towns that are connected via small trails or paved roads for humans, and rivers or lakes for mosquitoes, over counties to provinces and countries, or even on a global scale by transport via boats and air. This variability in geographic scale also affects judicious choices of the mobility matrices needed in our model: people travel far less frequently via air to other countries, than they do to the local fitness club two towns over. There is nothing singular about the problem of choosing patches in the context of the patched Ross-Macdonald model investigated here. In fact, users of any patch model face this issue as well. Nevertheless, we believe that they constitute a good first step towards a better understanding of more complicated models that incorporate spatial features more explicitly, such as partial differential equations models. 


\section{Appendices}

\section{A Quasi-monotone matrices and monotone sys- tems}

An interesting class of matrices are so-called quasi-monotone matrices ${ }^{3}$. They are real, $n \times n$ matrices $A$ such that:

$$
A_{i j} \geq 0 \text { if } i \neq j
$$

In other words, they have non-negative off-diagonal entries. For vectors and matrices alike, we use the order symbols $\leq,<$ and $<<$ to denote non-negativity, non-zero and non-negative, and positivity respectively. For instance $0<x$ means that $x$ is a nonzero, non-negative vector.

We collect some relevant properties of quasi-monotone matrices, which are consequences of the celebrated Perron-Frobenius Theorem, see for example [7]:

Theorem 6. Let $A$ be a quasi-monotone and irreducible matrix. Then there is a real and simple eigenvalue $s$ (called the stability modulus) with corresponding eigenvector $x>>0$ :

$$
A x=s x,
$$

and $\operatorname{Re}(\lambda)<s$ for every eigenvalue $\lambda$ of $A$, other than s. Moreover, every eigenvector $z>0$ of $A$ must be a scalar multiple of $x$.

Suppose that $A_{1}$ and $A_{2}$ are quasi-monotone, irreducible matrices with stability moduli $s_{1}$ and $s_{2}$. If $A_{1}<A_{2}$, then $s_{1}<s_{2}$. If $A_{1}$ and $A_{2}$ are only quasi-montone, and $A_{1} \leq A_{2}$, then $s_{1} \leq s_{2}$.

We will also need the following result concerning a specific non-negative matrix featured later:

Proposition 1. Let $A \geq 0$ and $B \geq 0$ be $n \times n$ matrices and let

$$
C=\left(\begin{array}{cc}
0 & A \\
B & 0
\end{array}\right)
$$

Then $C$ is irreducible if and only if both $A B$ and $B A$ are irreducible. If $C$ is irreducible, then $(R(C))^{2}=R(A B)=R(B A)$.

Proof. It is well-known that a non-negative matrix $X$ is irreducible if and only if there exists an integer $k>0$ such that $X+X^{2}+\cdots+X^{k}>>0$, see [7]. Note that if $m$ is a positive integer, then

$$
C+C^{2}+\cdots+C^{m}=\left\{\begin{array}{l}
\left(\begin{array}{cc}
\sum_{i=1}^{\frac{m-1}{2}}(A B)^{i} & A \sum_{i=0}^{\frac{m-1}{2}}(B A)^{i} \\
B \sum_{i=0}^{\frac{m-1}{2}}(A B)^{i} & \sum_{i=1}^{\frac{m-1}{2}}(B A)^{i}
\end{array}\right), \text { if } m \text { is odd } \\
\left(\begin{array}{cc}
\sum_{i=1}^{\frac{m}{2}}(A B)^{i} & A \sum_{i=0}^{\frac{m}{2}}-1 \\
B \sum_{i=0}^{\frac{m}{2}-1}(A B)^{i} & \sum_{i=1}^{\frac{m}{2}}(B A)^{i}
\end{array}\right), \text { if } m \text { is even }
\end{array}\right.
$$

\footnotetext{
${ }^{3}$ In the literature these are also known as Metzler matrices.
} 
If $C$ is irreducible, then there is some positive integer $k$, with necessarily $k>1$, such that the matrix $C+\cdots+C^{k}>>0$. Then the diagonal blocks in the expression above with $m=k$ are positive matrices, and this implies that $A B$ and $B A$ are irreducible.

Conversely, if $A B$ and $B A$ are irreducible, let $k=\max \left(k_{1}, k_{2}\right)$, where $k_{1}$ and $k_{2}$ are positive integers such that $\sum_{i=1}^{k_{1}}(A B)^{i}>>0$ and $\sum_{i=1}^{k_{2}}(B A)^{i}>>0$. Since $A B$ and $B A$ are irreducible, no row of $A$ or $B$ can consist of zeros only, and hence $A \sum_{i=1}^{k}(B A)^{i}>>0$ and $B \sum_{i=1}^{k}(A B)^{i}>>0$ as well. Setting $m=2(k+1)$ yields that $C+\cdots+C^{m}>>0$, and then $C$ is irreducible.

If $C$ is irreducible, then the Perron-Frobenius Theorem [7] implies that $R(C)$ is a positive eigenvalue of $C$, and it has a corresponding positive eigenvector. Hence, there exist vectors $x>>0$ and $y>>0$, not both zero, such that:

$$
C\left(\begin{array}{l}
x \\
y
\end{array}\right)=R(C)\left(\begin{array}{l}
x \\
y
\end{array}\right),
$$

or equivalently, that

$$
\begin{aligned}
& A y=R(C) x \\
& B x=R(C) y
\end{aligned}
$$

Then

$$
A B x=(R(C))^{2} x \text { and } B A y=(R(C))^{2} y .
$$

But $A B$ and $B A$ are irreducible (because $C$ is irreducible), and since $x>>0$ and $y>>0$, the Perron-Frobenius Theorem implies that

$$
R(A B)=(R(C))^{2}=R(B A),
$$

concluding the proof.

Finally, we consider systems for which the linearization of the vector field in any point is a quasi-monotone and possibly irreducible matrix. Such system are examples of what are known as (strongly) monotone systems [32] and they have the remarkable property that their solutions satisfy a particular comparison principle stated below.

More precisely, we consider

$$
\dot{x}=f(x), \quad x \in U \subset \mathbb{R}^{n},
$$

where $U$ is a convex region in $\mathbb{R}^{n}$ and $f$ is a $C^{1}$ vector field. Then the following comparison principle holds, see [32] for a proof:

Proposition 2. Assume that the Jacobian matrix $J(x):=D f(x)$ is quasi-monotone in $U$. Then system (40) is monotone, i.e. if $x_{0}$ and $y_{0}$ are initial conditions in $U$ with corresponding solutions $x(t), y(t)$ both defined on some positive interval $\mathcal{I}$, we have that:

$$
x_{0} \leq y_{0} \Rightarrow x(t) \leq y(t), \quad t \in \mathcal{I} .
$$

If $J(x)$ is quasi-monotone and irreducible in $U$, then the system is strongly monotone, i.e. it is monotone, and for initial conditions $x_{0} \leq y_{0}$, we have the following stronger property:

$$
x_{0}<y_{0} \Rightarrow x(t)<<y(t), \quad t \in \mathcal{I} \text {. }
$$




\section{B Proof of the dychotomy}

Proof of Theorem 2. System (19) - (20) is strongly monotone on $[0,1)^{2 n}$ by Proposition 2. Indeed, the Jacobian matrix is:

$$
J(x, y)=\left(\begin{array}{cc}
-D_{1}(y) & \operatorname{diag}(\mathbf{1}-x) \operatorname{diag}^{-1}(\rho) P \operatorname{diag}\left(\rho R_{0} r / \alpha\right) \\
\operatorname{diag}(\mathbf{1}-y) Q \operatorname{diag}(\mu \alpha) & -D_{2}(x)
\end{array}\right),
$$

where $D_{1}(y)$ and $D_{2}(x)$ are positive diagonal matrices whose diagonal entries depend only on the indicated arguments $y$ and $x$ respectively. As long as all $x_{i}$ and all $y_{i}$ are not equal to $1, J(x, y)$ is a quasi-monotone and irreducible matrix. (irreducibility follows from Proposition 1 because $P Q$ and $Q P$ are irreducible, and because no component of $x$ or $y$ equals 1 ) Consequently, the system is strongly monotone in this region of the state space.

Nonzero solutions starting on the boundary of $[0,1]^{2 n}$ enter $(0,1)^{2 n}$ instantaneously (when $x_{i}=1$ or $y_{i}=1$ this is immediate; and when $x_{i}=0$ or $y_{i}=0$, this follows because the flow is strongly monotone on $[0,1)^{2 n}$ and because $(x, y)=(0,0)$ is a steady state). In particular, the only steady state on the boundary of $[0,1]^{2 n}$ is the zero steady state $(x, y)=(0,0)$.

Next we consider the local stability properties of the zero steady state $(0,0)$. These are determined by the location of eigenvalues of $J(0,0)$ in the complex plane. Since $D_{1}(0)=\operatorname{diag}(r)$ and $D_{2}(0)=\operatorname{diag}(\mu)$, we have that:

$$
J(0,0)=\left(\begin{array}{cc}
-\operatorname{diag}(r) & \operatorname{diag}^{-1}(\rho) P \operatorname{diag}\left(\rho R_{0} r / \alpha\right) \\
Q \operatorname{diag}(\mu \alpha) & -\operatorname{diag}(\mu)
\end{array}\right)
$$

Following [13] we rewrite this matrix as the difference of a non-negative matrix $F$ and a nonsingular M-matrix $V$ as follows:

$$
J(0,0)=F-V
$$

where

$$
F=\left(\begin{array}{cc}
0 & \operatorname{diag}^{-1}(\rho) P \operatorname{diag}\left(\rho R_{0} r / \alpha\right) \\
Q \operatorname{diag}(\mu \alpha) & 0
\end{array}\right), \text { and } V=\left(\begin{array}{cc}
\operatorname{diag}(r) & 0 \\
0 & \operatorname{diag}(\mu)
\end{array}\right) .
$$

Let $s$ denote the stability modulus of $J(0,0)$. The proof of the Theorem 2 in [13] shows that:

$$
s\left\{\begin{array} { l } 
{ < 0 } \\
{ = 0 } \\
{ > 0 }
\end{array} \quad \text { if and only if } R ( F V ^ { - 1 } ) \left\{\begin{array}{l}
<1 \\
=1 \\
>1
\end{array}\right.\right.
$$

Consequently, the local stability properties of the steady state $(0,0)$ which are determined by the sign of $s$, can be equivalently derived from the spectral radius of the matrix $F V^{-1}$ :

$$
F V^{-1}=\left(\begin{array}{cc}
0 & \operatorname{diag}^{-1}(\rho) P \operatorname{diag}\left(\left(\rho R_{0} r\right) /(\alpha \mu)\right) \\
Q \operatorname{diag}(\alpha \mu / r) & 0
\end{array}\right) .
$$


It follows from Proposition 1 (note that the irreducibility condition in that Proposition is satisfied because both $P Q$ and $Q P$ are irreducible), and from the fact that $R(R S)=R(S R)$ for any two square matrices $R$ and $S$, that:

$$
\left(R\left(F V^{-1}\right)\right)^{2}=R(S)
$$

where the matrix $S$ is defined in (21). Thus it follows from (41) that the stability modulus of $J(0,0)$ is negative, zero or positive if and only if $R(S)$ is less than 1 , equal to 1 or larger than 1 respectively. Consequently, the zero steady state $(x, y)=(0,0)$ is locally asymptotically stable, respectively unstable if $R(S)$ is less than 1 , respectively larger than 1 .

We now distinguish two cases:

1. $R(S) \leq 1$. In this case we shall prove that the zero steady state $(x, y)=(0,0)$ is globally attractive, i.e. all solutions of (19) - (20) converge to it.

First we show that there is no other steady state in $[0,1]^{2 n}$. To see this, assume that there is a nonzero steady state $(\bar{x}, \bar{y})$, which must necessarily belong to $(0,1)^{2 n}$ (since we have shown that the only steady state on the boundary of $[0,1]^{2 n}$ is the zero steady state). Then the following steady state expression must hold:

$$
A(\bar{x}, \bar{y})\left(\begin{array}{l}
\bar{x} \\
\bar{y}
\end{array}\right)=\left(\begin{array}{l}
0 \\
0
\end{array}\right)
$$

where

$$
A(\bar{x}, \bar{y}):=\left(\begin{array}{cc}
-\operatorname{diag}(r) & \operatorname{diag}(1-\bar{x}) \operatorname{diag}^{-1}(\rho) P \operatorname{diag}\left(\rho R_{0} r / \alpha\right) \\
\operatorname{diag}(1-\bar{y}) Q \operatorname{diag}(\mu \alpha) & -\operatorname{diag}(\mu)
\end{array}\right)
$$

Note that $A(\bar{x}, \bar{y})$ is a quasi-monotone, irreducible matrix (irreducibility follows from Proposition 1 because $P Q$ and $Q P$ are irreducible, and because $(\bar{x}, \bar{y})$ belongs to $\left.(0,1)^{2 n}\right)$, and that

$$
A(\bar{x}, \bar{y})<J(0,0)
$$

Then Theorem 6 implies that

$$
s_{1}<s_{2}
$$

where $s_{1}$ is the stability modulus of $A(\bar{x}, \bar{y})$, and $s_{2}$ is the stability modulus of $J(0,0)$. But since $R(S) \leq 1$, it follows from what was said above that $s_{2} \leq 0$, and hence

$$
s_{1}<0
$$

But in view of (42), Theorem 6 implies that the stability modulus of $A(\bar{x}, \bar{y})$ is equal to 0 . Hence we have reached a contradiction, and we conclude that $(0,0)$ is the only steady state of system $(19)-(20)$ in $[0,1]^{2 n}$.

To finish the proof in this case we show next that all solutions converge to the zero steady state. Consider the initial condition $(\mathbf{1}, \mathbf{1})$, the North East corner of the state space $[0,1]^{2 n}$. Note that the vector field in this point is pointing to the South West:

$$
\left(\begin{array}{l}
\mathcal{F}(\mathbf{1}, \mathbf{1}) \\
\mathcal{G}(\mathbf{1}, \mathbf{1})
\end{array}\right)=\left(\begin{array}{l}
-r \\
-\mu
\end{array}\right)<<\left(\begin{array}{l}
0 \\
0
\end{array}\right)
$$


Here, $\left(\begin{array}{l}\mathcal{F}(x, y) \\ \mathcal{G}(x, y)\end{array}\right)$ denotes the vector field of system $(19)-(20)$. Then it follows that the solution starting in $(\mathbf{1}, \mathbf{1})$ is strictly decreasing with respect to the componentwise partial order on $[0,1]^{2 n}$. Since this solution is also bounded below by the zero steady state, it follows that this solution must converge to a steady state of the system. Since there is only one steady state, namely $(x, y)=(0,0)$, this solution converges to $(0,0)$. Finally, since the system is monotone, every solution starting in $[0,1]^{2 n}$ is bounded above by the solution starting in $(\mathbf{1}, \mathbf{1})$ by Proposition 2 , and hence all solutions converge to $(0,0)$ as well.

2. $R(S)>1$. In this case, the stability modulus of $J(0,0)$ is positive, and then a Taylor approximation of the vector field near the zero steady state $(x, y)=$ $(0,0)$, shows that

$$
\left(\begin{array}{l}
\mathcal{F}\left(\epsilon v_{1}, \epsilon v_{2}\right) \\
\mathcal{G}\left(\epsilon v_{1}, \epsilon v_{2}\right)
\end{array}\right)>>\left(\begin{array}{l}
0 \\
0
\end{array}\right)
$$

for all sufficiently small and positive $\epsilon$ where $\left(v_{1}, v_{2}\right)>>0$ is the eigenvector corresponding to the positive stability modulus of $J(0,0)$ (see Theorem $6)$. Then by the theory of monotone systems there exists a steady state $\left(\bar{x}_{m}, \bar{y}_{m}\right)>>0$ in $(0,1)^{2 n}$, and a connecting orbit between $(0,0)$ and $\left(\bar{x}_{m}, \bar{y}_{m}\right)$, converging to 0 in backward time and to $\left(\bar{x}_{m}, \bar{y}_{m}\right)$ in forward time, see Theorem 4.3.3 in [32]. Similarly, since (44) continues to hold in this case, there exists a steady state $\left(\bar{x}_{M}, \bar{y}_{M}\right)>0$ and a connecting orbit starting at $(\mathbf{1}, \mathbf{1})$ and converging to $\left(\bar{x}_{M}, \bar{y}_{M}\right)$ in forward time. We know that both $\left(\bar{x}_{m}, \bar{y}_{m}\right)$ and $\left(\bar{x}_{M}, \bar{y}_{M}\right)$ belong to $(0,1)^{n}$. Moreover, it can be shown that $\left(\bar{x}_{m}, \bar{y}_{m}\right) \leq$ $\left(\bar{x}_{M}, \bar{y}_{M}\right)$ (for otherwise, a comparison argument would violate the monotonicity of the system). The key step is to show that $\left(\bar{x}_{m}, \bar{y}_{m}\right)=\left(\bar{x}_{M}, \bar{y}_{M}\right)$. We argue by contradiction, and assume that they are different. Then in particular, there holds:

$$
\left(\bar{x}_{m}, \bar{y}_{m}\right)<\left(\bar{x}_{M}, \bar{y}_{M}\right) \text {. }
$$

Let $(\bar{x}, \bar{y})$ be an arbitrary steady state in $(0,1)^{2 n}$. The steady state equations can be written as in $(42)-(43)$, and then Theorem 6 implies that the stability modulus of $A(\bar{x}, \bar{y})$ is zero. Thus, both $A\left(\bar{x}_{m}, \bar{y}_{m}\right)$ and $A\left(\bar{x}_{M}, \bar{y}_{M}\right)$ have the same stability modulus, namely zero. However, if $\left(\bar{x}_{m}, \bar{y}_{m}\right)<\left(\bar{x}_{M}, \bar{y}_{m}\right)$, then the structure of the matrix $A(x, y)$ implies that $A\left(\bar{x}_{m}, \bar{y}_{m}\right)<A\left(\bar{x}_{M}, \bar{y}_{m}\right)$, and then Theorem 6 implies that the stability moduli of these matrices are different. We have reached a contradiction, and consequently, there is only one nonzero steady state in $[0,1]^{2 n}$. Finally, exploiting the strong monotonicity of the system, it follows that all nonzero solutions converge to this nonzero steady state by the comparison argument in Proposition 2. 


\section{References}

[1] Arino, J., and van den Driessche, P., A multi-city epidemic model, Mathematical Population Studies 10, 175-193, 2003.

[2] Arino, J., Davis, J.R., Hartley, D., Jordan, R., Miller, J.M., and van den Driessche, P., A multi-species epidemic model with spatial dynamics, Mathematical Medicine and Biology 22, 129-142, 2005.

[3] Arino, J., Diseases in metapopulations, In: Ma, Z., Zhou, Y., and Wu, J., Eds., Modeling and Dynamics of Infectious Diseases, 65-123, World Scientific, 2009 .

[4] Arino, J., and Portet, S., Epidemiological implications of mobility between a large urban centre and smaller satellite cities, Journal of Mathematical Biology 71, 1243-1265, 2015.

[5] Auger, P., Kouokam, E., Sallet, G., Tchuente, M., and Tsanou, B., The RossMacdonald model in a patchy environment, Mathematical Biosciences 216, 123-131, 2008.

[6] Bejon, P., Williams, T.N., Nyundo,C., Hay, S.I., Benz, D., Gething,P.W., Otiende, M., Peshu, J., Bashraheil,M., Greenhouse,B., Bousema, T., Bauni, E., Marsh,K., Smith, D.L., and Borrmann, S., A micro-epidemiological analysis of febrile malaria in Coastal Kenya showing hotspots within hotspots. eLife 1-19, doi:10.7554/eLife.02130, 2014.

[7] Berman, A., and Plemmons, P.J., Nonnegative Matrices in the Mathematical Sciences, SIAM, 1994.

[8] Bousema, T., Drakeley, C., Gesase, S., Hashim, R., Magesa, S., Mosha, F., Otieno, S., Carneiro, I., Cox, J., Msuya, E., Kleinschmidt, I., Maxwell, C., Greenwood, B., Riley, E., Sauerwein, R., Chandramohan, D., and Gosling, R., Identification of hot spots of malaria transmission for targeted malaria control, Journal of Infectious Diseases 201, 1764-1774, 2010.

[9] Bousema, T., Griffin, J.T., Sauerwein, R.W., Smith, D.L., Churcher, T.S., Takken, W., Ghani, A., Drakeley, C., and Gosling, R., Hitting hotspots: Spatial targeting of malaria for control and elimination, PLOS Medicine 9, e1001165, 2012.

[10] http://www.cdc.gov/malaria/about/faqs.html

[11] Cosner, C., Beier, J.C., Cantrell, R.S., Impoinvil, D., Kapitanski, L., Potts M.D., Troyo, A., and Ruan, S., The effects of human movement on the persistence of vector-borne diseases, Journal of Theoretical Biology 258, 550-560, 2009 .

[12] Cosner, C., Models for the effects of host movement in vector-borne disease systems, Mathematical Biosciences 270, 192-197, 2015. 
[13] van den Driessche, P., and Watmough, J. Reproduction numbers and subthreshold endemic equilibria for compartmental models of disease transmission, Mathematical Biosciences 180, 29-48, 2002.

[14] Gao, D., and Ruan, S., An SIS patch model with variable transmission coefficients, Mathematical Biosciences 232, 110-115, 2011.

[15] Gao, D., and Ruan, S., Malaria models with spatial effects, In: Chen, D., Moulin, B., and Wu, J., Eds., Analyzing and Modeling Spatial and Temporal Dynamics of Infectious Diseases, 3-30, John Wiley \& Sons, 2014.

[16] Githeko, A.K., Ayisi, J.M., Odada, P.K., Atieli, F.K., Ndenga, B.A., Githure, J.I., and Yan, G., Topography and malaria transmission heterogeneity in western Kenya highlands: prospects for focal vector control, Malaria Journal 5, 107, 2006.

[17] Hasibeder, G., and Dye, C., Population dynamics of mosquito-borne disease: persistence in a completely heterogeneous environment, Theoretical Population Biology 33, 31-53, 1988.

[18] Hsieh, Y.-H., van den Driessche, P., and Wang, L., Impact of Travel Between Patches for Spatial Spread of Disease, Bulletin of Mathematical Biology 69, 1355-1375, 2007.

[19] Killeen, G.F., Knols, B.G.J., and Gu, W., Taking malaria transmission out of the bottle: implications of mosquito dispersal for vector-control interventions, Lancet Infectious Diseases 3, 297-303, 2003.

[20] Koella, J.C., On the use of mathematical models of malaria transmission, Acta Tropica 49, 1-25, 1991.

[21] Lajmanovich, A., Yorke, J.A., A deterministic model for gonorrhea in a nonhomogeneous population. Mathematical Biosciences 28, 221-236, 1976.

[22] Li, C.-K., and Schneider, H., Journal of Mathematical Biology 44, 450-462, 2002.

[23] Manore, C.A., Hickmann, K.S., Hyman, J.M., Foppa, I.M., Davis, J.K., Wesson, D.M., and Mores, C.N., A network-patch methodology for adapting agent-based models for directly transmitted disease to mosquito-borne disease, arXiv:1405.2258v1.

[24] Mbogo, C.M., Mwangangi, J.M., Nzovu, J., Gu, W., Yan, G., Gunter, J.T., Swalm, C., Keating, J., Regens, J.L., Shililu, J.I., Githure, J.I., and Beier, J.C., Spatial and temporal heterogeneity of Anopheles mosquitoes and Plasmodium falciparum transmission along the Kenyan coast, American Journal of Tropical Medicine and Hygiene 68, 734-742, 2003.

[25] Moonen, B., Cohen, J.M., Snow, R.W., Slutsker, L., Drakeley, C., Smith, D.L., Abeyasinghe R.R., Rodriguez, M.H., Maharaj, R., Tanner, M., and Targett, G., Operation strategies to achieve and maintain malaria elimination, Lancet 376, 1692-1603, 2010. 
[26] Ruktanonchai, N.W., De Leenheer, P., Tatem, A.J., Alegana, V.A., Caughlin, T.T., zu Erbach-Schoenberg, E. , Loureno, C., Ruktanonchai, C.W., and Smith, D.L., Identifying Malaria Transmission Foci for Elimination Using Human Mobility Data, PLOS Computational Biology 12(4): e1004846. doi:10.1371/journal. pcbi.1004846.

[27] Paull, S.H., Song, S., McClure, K.M., Sackett, L.C., Kilpatrick A.M., and Johnson, P.T.J., From superspreaders to disease hotspots: linking transmission across hosts and space, Frontiers of Ecology and Environment 10, 75-82, 2012 .

[28] Prothero, R.M., Disease and mobility: a neglected factor in epidemiology, International Journal of Epidemiology 6, 259-267, 1977.

[29] Prosper, O., Ruktanonchai, N., and Martcheva, M., Assessing the role of spatial heterogeneity and human movement in malaria dynamics and control, Journal of Theoretical Biology 303, 1-14, 2012.

[30] Rodriguez, D.J., and Torres-Sorando, L., Models of infectious diseases in spatially heterogeneous environments, Bulletin of Mathematical Biology 63, 547$571,2001$.

[31] Ruktanonchai, N.W., De Leenheer, P., Tatem, A.J., Alegana, V.A., Caughlin, T.T., zu Erbach-Schoenberg, E., Lourenco, C., Ruktanonchai, C.W., and Smith, D.L., Identifying Malaria Transmission Foci for Elimination Using Human Mobility Data. PLoS Comput Biol 12(4): e1004846. doi:10.1371/journal. pcbi. 1004846 .

[32] Smith, H.L., Monotone Dynamical Systems, AMS, 1995.

[33] Smith, D.L., and McKenzie, F.E., Statics and dynamics of malaria infection in Anopheles mosquitoes, Malaria Journal 3,13, 2004.

[34] Smith, D.L., Battle, K.E., Hay, S.I., Barker, C.M., Scott, T.W., and McKenzie, F.E., Ross, Macdonald, and a Theory for the Dynamics and Control of Mosquito-Transmitted Pathogens, PLoS Pathogens 8, e1002588, 2012.

[35] Stoddard, S.T., Morrison, A.C., Vazquez-Prokopec, G.M., Soldan V.P., Kochel, T.J., Kitron, U., Elder, J.P., and Scott, T.W., The role of human movement in the transmission of vector-borne pathogens, PLOS Neglected Tropical Diseases 3, e481, 2009.

[36] Tatem, A.J., and Smith, D.L., International population movements and regional Plasmodium falciparum malaria elimination strategies, Proceedings of the National Academy of Sciences 107, 12222-12227, 2010.

[37] Wang, W., Epidemic models with population dispersal, In: Takeuchi,Y., Iwasa,Y., and Sato, K., Eds., Mathematics for Life Science and Medicine, 67-95, Springer-Verlag, 2007. 
[38] Wesolowski A., Eagle, N., Tatem, A.J., Smith, D.L., Noor, A.M., Snow, R.W., and Buckee C.O., Quantifying the impact of human mobility on malaria, Science 338, 267-270, 2012.

[39] Zhuo, G., Munga, S., Minakawa, N., Githeko, A.K., and Yan, G., Spatial relationship between adult malaria vector abundance and environmental factors in western Kenya highlands, American Journal of Tropical Medicine and Hygiene 77, 29-35, 2007. 